\title{
Propuesta de programación para la construcción del CPL Esmeraldas mediante el Método de la Cadena de Trabajo
}

\author{
Ing. Jorge José Arroyo Orozco, M.Sc. ${ }^{1}$, Paula Silvana Alarcón Segura ${ }^{1}$, Ing. Gino Fernando Flor Chávez ${ }^{1}$ e Ing. \\ Natalia Jaquelin Chamorro Enríquez ${ }^{1}$ \\ ${ }^{1}$ Universidad de Guayaquil, Ecuador. \\ jorge.arroyoo@ug.edu.ec, paula.alarcons@ug.edu.ec, gino.florch@ug.edu.ec
}

\begin{abstract}
Resumen- Esta investigación propone una programación o planificación para la construcción de la estructura de hormigón armado de los pabellones de mínima seguridad del Centro de Privación de Libertad en la Ciudad de Esmeraldas. Para llegar a una programación óptima se deben analizar todos los factores que intervienen al momento de la construcción, como lo son; el presupuesto final de la obra, el tiempo de ejecución del proyecto, los recursos necesarios para el desarrollo del mismo y los costos indirectos. El análisis se lo realizó con el método de la cadena de trabajo, este método se divide en tres; método sucesivo, paralelo y en cadena, en base a los resultados, se escoge el método apropiado para la construcción del Centro de Privación de Libertad de Esmeraldas, considerando el más conveniente tanto en costos como en tiempo de ejecución de la obra.
\end{abstract}

Palabras Claves-- Programación de obra, tiempos de construcción, recursos de obra, cadena de trabajo.

Abstract- This research proposes a programming or planning for the construction of the reinforced concrete structure of the minimum-security pavilions of the deprivation of freedom center in the city of Esmeraldas. To achieve an optimal programming, all the factors that intervene at the time of construction must be analyzed, as they are; the final budget of the work, the time of execution of the project, the necessary resources for its development and the indirect costs. The analysis was done with the chain of work method, this method is divided into three; successive, parallel and chain method, based on the results, the appropriate method is chosen for the construction of the deprivation of freedom center of the city of Esmeraldas, considering the most suitable in terms of costs and time of execution of the work.

Keywords-- Work programming, construction times, work resources, work chain.

\section{INTRODUCCIÓN}

Una programación de obra busca la secuencia de actividades, en función de los recursos disponibles, en base a esto se establece un periodo de tiempo para cada actividad [1], [2], [3], [4].

Los métodos de construcción se han ido desarrollando con el paso del tiempo, con la finalidad de que la obra se ejecute de forma rápida, sin olvidar, el costo de la obra y la calidad de los materiales [5], [6].

Digital Object Identifier (DOI):

http://dx.doi.org/10.18687/LACCEI2019.1.1.379 ISBN:

978-0-9993443-6-1 ISSN: 2414-6390
En la presente investigación se propuso una programación óptima en las actividades para las estructuras de hormigón armado en la obra de los pabellones de mínima seguridad en el CPL (Centro de Privación de Libertad) de la ciudad de Esmeraldas.

El método que se utilizó fue el de la cadena de trabajo, que consiste en; asemejar los trabajos, conectar los procesos de construcción y optimizar recursos, con esto se avanza de manera rápida y el valor final del proyecto se reduce [5] ,[6], [7].

La programación en cadena consta de dos periodos; el periodo lento, que se basa en una velocidad de producción determinada, del número de trabajadores, calidad y conjunto de maquinaria empleadas en la construcción. El segundo periodo es el acelerado, este proceso pretende elaborar los trabajos en tiempos sumamente cortos, de forma razonable, mediante la sincronización de trabajos en el tiempo establecido, empleando el mayor número de trabajadores posibles en la obra civil y la adquisición de los materiales y máquinas necesarias para cumplir con el proyecto [5], [6], [7].

En la planificación por medio de la cadena de trabajo existen tres métodos; método sucesivo, método paralelo y método en cadena. El método sucesivo comprende en realizar las actividades por partes, es decir, al terminar un trabajo se inicia el siguiente. En este método los plazos de trabajo son largos y se utiliza el mínimo de los recursos. Por otro lado, el método paralelo o simultáneo como su nombre lo indica, consiste en realizar las actividades de forma paralela en un tiempo determinado, incrementando los recursos de la obra. Por último, el método en cadena o en serie se desenvuelve usando cualidades de los procesos anteriores, es decir, con actividades similares se usará el método sucesivo y con las actividades distintas se usará el método paralelo, de esta manera de optimiza recursos e incrementa la productividad [5], $[6],[7]$.

En una obra civil se tendrá divisiones de trabajo (subproyectos), estos pueden ser desglosados en trabajos más específicos, alcanzando el correcto desarrollo del mismo [1], [2], [5], [6], [8], [9]. En esta investigación se programó las actividades para la cimentación de los Pabellones de Mínima Seguridad, dividiendo las tareas en: excavación de zanjas, 
concreto de zapata, encofrado de contrapiso, concreto de contrapiso y relleno con material propio.

\section{MetodologíA}

Ref. [1] establece los siguientes pasos para elaborar una programación de obra. En este caso, se ha agregado los tres métodos mencionados anteriormente, para los trabajos de estructura de hormigón armado de los Pabellones de mínima seguridad:

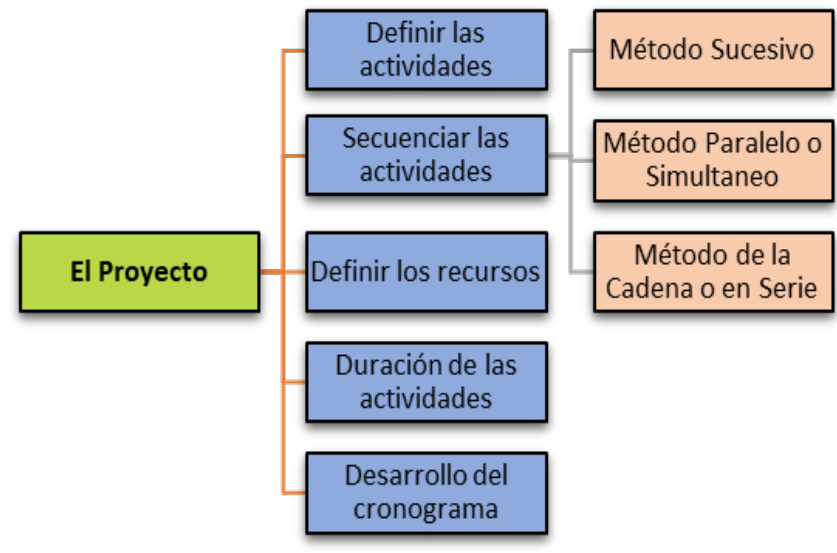

\section{A. Presupuesto}

En la ingeniería civil, es la estimación del valor total de una obra, este valor se divide en dos; costos directos y costos indirectos. Los costos directos incluyen; la mano de obra, materiales, transporte y equipos. Los costos indirectos contienen; gastos de oficina o campamento, salario de profesionales, etc., los costos indirectos varían según la localidad de la obra y su alcance [3], [4], [8], [9].

En la elaboración del presupuesto, como primer paso, se deben conocer las cantidades de obra, estas fueron obtenidas de los planos estructurales del proyecto, enfocándose únicamente en los Pabellones de mínima seguridad, que están conformados por; planta baja, dos niveles altos y una losa de cubierta [6].

La investigación se realizó en 4 Pabellones de varones, cada pabellón contiene; 17 unidades de vivienda más 2 discapacitados en la planta baja, 22 unidades de vivienda en el piso 1 y 2 [6].

\section{B. Caso 1: Presupuesto con el método sucesivo}

Al seguir los pasos de la metodología, el segundo ítem se lo desarrolla con el método sucesivo al secuenciar las actividades, con esto se obtiene un presupuesto calculado en base a una programación de obra con método sucesivo, que se lo detalla en el anexo, Tabla I. Con la siguiente ecuación se puede determinar la duración de la obra con el método sucesivo:

$$
\mathrm{T}=\mathrm{m} \times \mathrm{Tc}
$$

Donde $T$ es el tiempo de construcción, Tc es la duración de la construcción de 1 pabellón y $m$ es el número de pabellones [5], [7].

\section{Caso 2: Presupuesto con el método paralelo o simultáneo}

Al seguir los pasos de la metodología, el segundo ítem se lo desarrolla con el método paralelo o simultáneo al secuenciar las actividades, con esto se obtiene un presupuesto calculado en base a una programación de obra con método paralelo, que se lo detalla en el anexo, Tabla II. En este caso, el tiempo total de la obra será igual al tiempo que dure uno de los trabajos:

$$
\mathrm{T}=\mathrm{Tc}
$$

Donde $T$ es el tiempo de construcción, Tc es la duración de la construcción de 1 pabellón [5], [7].

\section{Caso 3: Presupuesto con el método en cadena o en serie}

Al seguir los pasos de la metodología, el segundo ítem se lo desarrolla con el método en cadena o en serie al secuenciar las actividades, con esto se obtiene un presupuesto calculado en base a una programación de obra con método en cadena, que se lo detalla en el anexo, Tabla III. El tiempo que dure la construcción debe cumplir la siguiente condición:

$$
\mathrm{T}<\mathrm{mx} \mathrm{Tc}
$$

Donde $T$ es el tiempo de construcción, $T c$ es la duración de la construcción de 1 pabellón $\mathrm{y} m$ es el número de pabellones [5], [7].

\section{ANÁLISIS DE RESULTADOS}

A. Comparación de los tiempos de trabajo realizando la programación de la obra con los tres métodos de la cadena de trabajo

Al realizar un cronograma de obra con cada método de la cadena de trabajo se pudo apreciar la variación en los tiempos que puede durar la obra según el método [6].

Los cronogramas elaborados en el programa Microsoft Project por los métodos; sucesivo, paralelo y en cadena, dieron como resultado duraciones de; 480 días, 120 días y 300 días, como se puede observar en el anexo fig. 1, 2 y 3 , respectivamente.

En las fig. 4, 5 y 6, se puede entender de forma representativa el porqué de los tiempos, para comprender mejor cada método. En el método sucesivo se plantea construir 1 pabellón a la vez (fig. 4), lo que extiende el plazo de la obra a 480 días, por su parte, el método paralelo planea construir los 4 pabellones al mismo tiempo (fig. 5), reduciendo radicalmente el tiempo de construcción a 120 días, finalmente, se tiene el método en cadena o en serie que combina los métodos anteriores, enlazando o traslapando la construcción de un pabellón con el siguiente (fig. 6), trayendo como resultado una duración de obra de 300 días. 


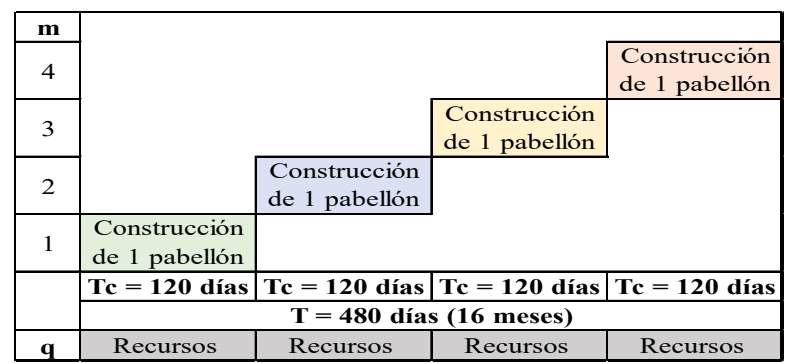

Fig. 4 Cronograma representativo de la construcción de los 4 pabellones por medio del método sucesivo.

Fuente: Elaboración propia.

\begin{tabular}{|c|c|}
\hline $\mathbf{m}$ & \\
\hline 4 & Construcción de 1 pabellón \\
\hline 3 & Construcción de 1 pabellón \\
\hline 2 & Construcción de 1 pabellón \\
\hline 1 & Construcción de 1 pabellón \\
\hline & $\mathbf{T c}=\mathbf{1 2 0}$ días \\
\cline { 2 - 2 } & $\mathbf{T 2 0}$ días (4 meses) \\
\hline $\mathbf{q}$ & Recursos \\
\hline
\end{tabular}

Fig. 5 Cronograma representativo de la construcción de los 4 pabellones por medio del método paralelo o simultáneo.

Fuente: Elaboración propia.

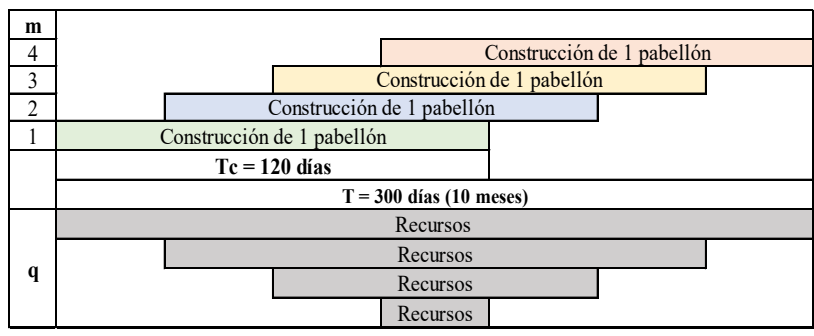

Fig. 6 Cronograma representativo de la construcción de los 4 pabellones por medio del método en cadena o en serie.

Fuente: Elaboración propia.

B. Comparación del costo del recurso de obra: encofrado FORSA, con los tres métodos de la cadena de trabajo

Considerando que este recurso es el más importante del proyecto por su inversión inicial y dado que en función de este, se determinó los plazos de ejecución en los tres métodos y para poder cumplir con los plazos anteriormente propuestos, se consideró la gestión de adquisición de los siguientes juegos de encofrados:

1) Método Sucesivo: Se propuso adquirir 1 juego de encofrado completo para la ejecución de 1 pabellón a la vez, valorado en USD \$ $311,793.65$, el cual sería utilizado de manera sucesiva en la ejecución de los 4 pabellones, concluyendo la estructura en un plazo de 480 días.
2) Método Paralelo: Se consideró la adquisición de 4 juegos de encofrados valorado en USD $\$ 1,248,084.91$, lo cual garantizaría que se ejecuten los 4 pabellones a la vez, disminuyendo el plazo a 120 días.

3) Método en Cadena: Se optimizó con la adquisición de los módulos de encofrado, invirtiendo USD \$ 624,042.46 en 2 juegos de encofrados, garantizando una ejecución en un plazo de 300 días, compartiendo de forma sucesiva los encofrados entre dos pabellones a la vez.

Con los resultados de los casos 1, 2 y 3, mencionados anteriormente, se obtuvo el costo total de la obra para los tres métodos de la cadena de trabajo (fig. 7), con estos costos y el monto total que se invertiría por juego de encofrado en cada método se logra hacer una comparación. En la fig. 8 se puede observar los porcentajes que representa el encofrado según el método, siendo estos; $12.95 \%, 37.73 \%$ y $23.09 \%$, del monto total de la obra, para el 1 juego, 4 juegos y 2 juegos de encofrados, respectivamente.

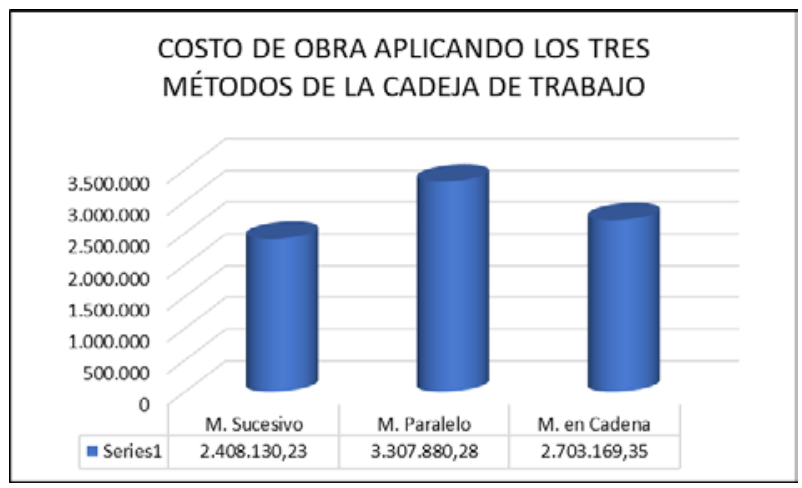

Fig. 7 Diagrama comparativo del costo total de la obra por los tres métodos propuestos.

Fuente: Elaboración propia.

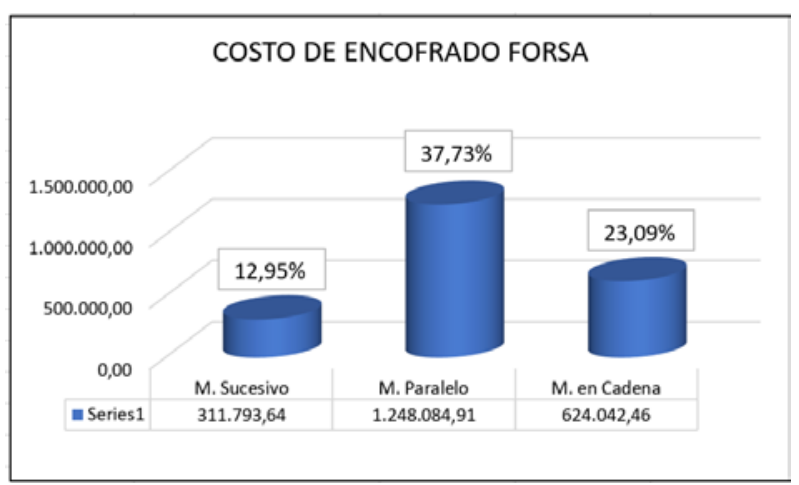

Fig. 8 Diagrama comparativo del costo del encofrado FORSA, en cada método de la cadena trabajo, aplicando los juegos de encofrados propuestos. Fuente: Elaboración propia.

C. Comparación de costos indirectos de la obra, con los tres métodos de la cadena de trabajo

En función de la cantidad de encofrados por adquirir en cada uno de los métodos, y a partir de ello, estableciendo los plazos de ejecución de la estructura de hormigón armado de 
los pabellones de mínima seguridad, se calculan los costos indirectos (indirectos de campo, indirectos de operación y utilidad), quedando de la siguiente manera:

En el Método Sucesivo con un plazo de 480 días se obtuvo un porcentaje de costos indirectos del $29 \%$, este es el porcentaje más elevado debido a su largo plazo de ejecución de obra, se aumentan los meses de pago al personal, adquisición de transporte, etc., así mismo para el Método Paralelo con un plazo de 120 días se llegó a un porcentaje de indirectos del $18 \%$; obviamente al disminuir el plazo de entrega de la obra también disminuye la incidencia de los costos indirectos en el proyecto, posteriormente para el Método en Cadena, con dos juegos de encofrados y con un plazo de ejecución de 300 días se calculó un porcentaje de indirectos del $24 \%$, siendo la media de los costos indirectos de los métodos anteriormente mencionados. En la fig. 9 se puede apreciar los resultados en base a los tres métodos; precio final de la obra, tiempo de ejecución del proyecto y sus respectivos costos indirectos.

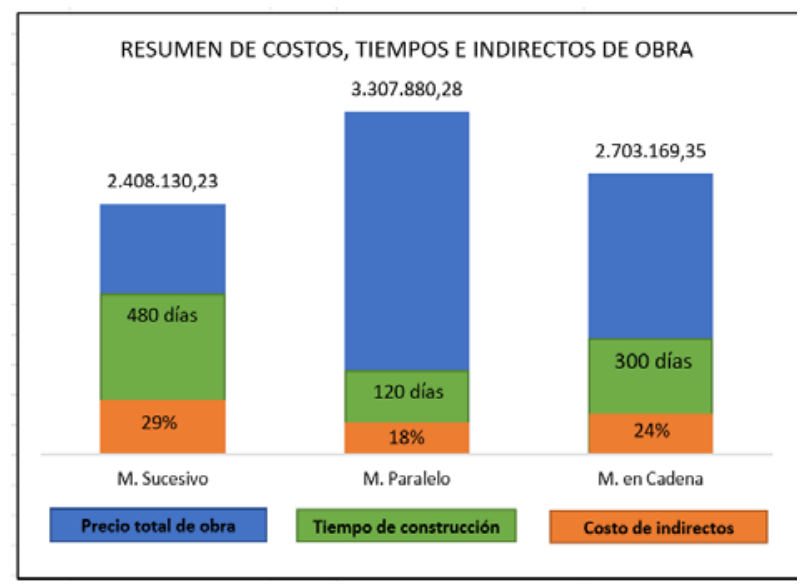

Fig. 9 Diagrama comparativo del costo total de la obra, tiempo de construcción y costo indirecto de obra por cada método de la cadena de trabajo. Fuente: Elaboración propia.

\section{CONCLUSIONES}

Mediante el análisis realizado por los tres métodos de la cadena de trabajo, se logró conocer el presupuesto final del proyecto y su tiempo de construcción según el método.

En el método sucesivo se llegó a un presupuesto de $\$ 2^{\prime} 408.130,23$ en un tiempo de ejecución de 480 días (16 meses), siendo este el más económico en comparación con los otros dos métodos. En lo que respecta al método paralelo se obtuvo un presupuesto de $\$ 33^{\prime} 307.880,28$ y un tiempo de construcción de 120 días (4 meses), en el tema de plazos este sería el más apropiado por su corta duración, pero por costos resulta el más caro. Por último, en el método en cadena o en serie se calculó un presupuesto de $\$ 2^{`} 703.169,35$ con un periodo de ejecución de 300 días (10 meses), alcanzando el punto de equilibrio tanto en costos, porcentaje de indirectos como en tiempos de ejecución, entre ambos métodos.
Si bien se determina que el método idóneo en función de la comparación realizada anteriormente es el método en cadena, cabe recalcar que la selección final depende de los tomadores de decisiones de las organizaciones como empresas constructoras, o entidades públicas, quienes son los que validan si desean prevalecer tiempo sobre inversión monetaria o al contrario.

Se puede observar que el método idóneo para estos trabajos donde existen construcciones lineales, como viviendas, departamentos, cárceles, hoteles, carreteras, etc., es el método en cadena, proporcionando un periodo de construcción razonable acorde a su presupuesto.

\section{REFERENCIAS}

[1] Project Management Institute, La guía de los fundamentos para la dirección de proyectos (Guía del PMBOK), Pensilvania: Project Management Institute, 2017.

[2] B. Cooke y P. Williams, Construction Planning Programming and Control, Inglaterra: PALGRAVE, 1998.

[3] Project Management Institute, Extensión de la construcción a la guía del PMBOK, Pensilvania: Project Management Institute, 2003.

[4] A. Mattos, Métodos de Planificación y Control de Obras del diagrama de barras al BIM, Barcelona: Reverte, 2014.

[5] W. Rodríguez, Gerencia de Construcción y del Tiempo-Costo, Lima: Empresa Editora Macro E.I.R.L., 2013.

[6] N. Chamorro, "Programación para la ejecución de los trabajos de estructuras de hormigón armado de los pabellones de mínima seguridad del CPL Esmeraldas mediante el Método de Cadena de Trabajo", tesis de grado, UG, Guayaquil, 2018.

[7] M. Budnikov, Fundamentos de la Construcción en Cadena, Moscú: Literatura de Construcción y Arquitectura de la RSSU, 1961.

[8] C. Suarez, Costo y tiempo en Edificación, México D.F.: Limusa, 2013.

[9] J. Olmedo, Planeación, Programación y Control de obras de Construcción, Ibagué: Alfaomega, 2016.
ORCID iD Ing. Jorge José Arroyo Orozco, M.Sc. https://orcid.org/0000-0002-4785-368X

ORCID iD Paula Silvana Alarcón Segura

https://orcid.org/0000-0002-9975-4157 


\section{ANEXOS}

TABLA I. PRESUPUESTO DE LA ESTRUCTURA DE HORMIGÓN ARMADO DE LOS 4 PABELLONES CON EL MÉTODO SUCESIVO.

Fuente: [6].

\begin{tabular}{|c|c|c|c|c|c|c|c|}
\hline RUBRO & DESCRIPCION & UNIDAD & CANTIDAD & $\begin{array}{l}\text { PRECIO } \\
\text { UNITARIO }\end{array}$ & $\begin{array}{l}\text { PRECIO } \\
\text { TOTAL }\end{array}$ & \begin{tabular}{|c|} 
CANTIDAD 1 \\
PABELLON \\
(ALA 1 Y ALA 2)
\end{tabular} & $\begin{array}{l}\text { NUMERO } \\
\text { DE DIAS }\end{array}$ \\
\hline 1 & EXCAVACION SIN CLASIFICAR & M3 & $2.298,37$ & 1,94 & $4.458,83$ & 574,59 & 2 \\
\hline 2 & DESALOJO DE LAS EXCAVACIONES DISTANCIA $10 \mathrm{KM}$ & M3-KM & $22.983,68$ & 0,50 & $11.491,84$ & $5.745,92$ & 2 \\
\hline 3 & RELLENO CON MATERIAL DE MEJORAMIENTO (INCLUIDO TRANSPORTE) & M3 & $1.189,74$ & 16,38 & $19.488,00$ & 297,44 & 1 \\
\hline 4 & RELLENO CON SUELO CEMENTO (INCLUIDO TRANSPORTE) & $\mathrm{M} 3$ & 320,54 & 33,59 & $10.767,07$ & 80,14 & 1 \\
\hline 5 & EMPEDRADO CON PIEDRA BOLA $(E=12 \mathrm{CM})$ & M3 & 85,42 & 37,38 & $3.193,03$ & 21,36 & 1 \\
\hline 6 & CAMA DE ARENA (2 CM) & M3 & 14,24 & 34,77 & 495,01 & 3,56 & 1 \\
\hline 7 & HORMIGON DE REPLANTILLO FC $=140 \mathrm{KG} / \mathrm{CM} 2 \mathrm{E}=5 \mathrm{CM}$ & M3 & 148,45 & 183,54 & $27.246,15$ & 37,11 & 7 \\
\hline 9 & ENCOFRADO DE ZAPATAS & M2 & 954,84 & 28,02 & $26.754,62$ & 238,71 & 10 \\
\hline 10 & ACERO DE REFUERZO EN BARRAS FY=4200 KG/CM2 (ZAPATAS) & KG & $47.984,03$ & 1,79 & $85.891,42$ & $11.996,01$ & 15 \\
\hline 11 & HORMIGON DE MUROS FC $=210 \mathrm{KG} / \mathrm{CM} 2$ & M3 & $1.242,97$ & 223,70 & $278.052,84$ & 310,74 & 21 \\
\hline 12 & ENCOFRADO DE MUROS & M2 & $34.504,88$ & 16,28 & $561.739,45$ & $8.626,22$ & 28 \\
\hline 13 & ACERO DE REFUERZO EN BARRAS FY=4200 KG/CM2 (MUROS) & KG & $157.891,36$ & 1,75 & $276.309,89$ & $39.472,84$ & 22 \\
\hline 14 & HORMIGON DE VIGAS DE ENTREPISO FC $=210 \mathrm{KG} / \mathrm{CM} 2$ & $\mathrm{M} 3$ & 265,73 & 238,77 & $63.447,87$ & 66,43 & 13 \\
\hline 15 & ENCOFRADO DE VIGAS DE ENTREPISO & $\mathrm{M} 2$ & $1.044,00$ & 18,20 & $19.000,80$ & 261,00 & 11 \\
\hline 16 & ACERO DE REFUERZO EN BARRAS FY=4200 KG/CM2 (VIGAS ENTREPISO) & KG & $50.235,92$ & 1,79 & $89.922,30$ & $12.558,98$ & 16 \\
\hline 17 & HORMIGON DE LOSAS DE ENTREPISO FC $=210 \mathrm{KG} / \mathrm{CM} 2$ & $\mathrm{M} 3$ & 672,97 & 226,18 & $152.212,81$ & 168,24 & 14 \\
\hline 18 & ENCOFRADO DE LOSAS DE ENTREPISO & M2 & $4.435,28$ & 16,80 & $74.512,70$ & $1.108,82$ & 12 \\
\hline 21 & ENCOFRADO DE LOSAS DE CAMASTROS & M2 & $2.817,72$ & 16,24 & $45.759,77$ & 704,43 & 7 \\
\hline 22 & ACERO DE REFUERZO EN BARRAS FY=4200 KG/CM2 (CAMASTROS) & KG & $21.097,44$ & 1,82 & $38.397,34$ & $5.274,36$ & 7 \\
\hline 23 & HORMIGON DE CONTRAPISO FC=210 KG/CM2 & M3 & 106,74 & 217,88 & $23.256,51$ & 26,69 & 2 \\
\hline 24 & MALLA ELECTROSOLDADA R-131 (5 MM / 150 MM) PARA CONTRAPISO & M2 & 711,60 & 7,86 & $5.593,18$ & 177,90 & 2 \\
\hline 25 & POLIETILENO & M2 & 711,84 & 2,68 & $1.907,73$ & 177,96 & 2 \\
\hline 26 & HORMIGON DE MURO DE CERRAMIENTO FC $=210 \mathrm{KG} / \mathrm{CM} 2$ & M3 & 526,80 & 270,94 & $142.731,19$ & 131,70 & 26 \\
\hline 27 & ENCOFRADO DE MUROS DE CERRAMIENTO & $\mathrm{M} 2$ & $2.715,44$ & 20,95 & $56.888,47$ & 678,86 & 28 \\
\hline \multirow[t]{2}{*}{28} & ACERO DE REFUERZO EN BARRAS FY=4200 KG/CM2 (MUROS CERRAMIENTO) & KG & $37.528,92$ & 1,83 & $68.677,92$ & $9.382,23$ & 23 \\
\hline & & & & & $2.408 .130,23$ & & \\
\hline
\end{tabular}

TABLA II. PRESUPUESTO de LA ESTRUCTURA DE HORMIGÓN ARMADO DE LOS 4 PABELLONES CON EL MÉTOdo PARALELO o SiMULTÁNEO. Fuente: [6].

\begin{tabular}{|c|c|c|c|c|c|c|c|}
\hline RUBRO & DESCRIPCION & UNIDAD & CANTIDAD & $\begin{array}{l}\text { PRECIO } \\
\text { UNITARIO }\end{array}$ & $\begin{array}{l}\text { PRECIO } \\
\text { TOTAL }\end{array}$ & \begin{tabular}{|c|} 
CANTIDAD 1 \\
PABELLON \\
(ALA 1 Y ALA 2)
\end{tabular} & $\begin{array}{c}\text { NUMERO } \\
\text { DE DIAS }\end{array}$ \\
\hline 1 & EXCAVACION SIN CLASIFICAR & M3 & $2.298,37$ & 1,77 & $4.068,11$ & 574,59 & 2 \\
\hline 2 & DESALOJO DE LAS EXCAVACIONES DISTANCIA $10 \mathrm{KM}$ & M3-KM & $22.983,68$ & 0,46 & $10.572,49$ & $5.745,92$ & 2 \\
\hline 3 & RELLENO CON MATERIAL DE MEJORAMIENTO (INCLUIDO TRANSPORTE) & M3 & $1.189,74$ & 14,99 & $17.834,26$ & 297,44 & 1 \\
\hline 4 & RELLENO CON SUELO CEMENTO (INCLUIDO TRANSPORTE) & M3 & 320,54 & 30,73 & $9.850,32$ & 80,14 & 1 \\
\hline 5 & EMPEDRADO CON PIEDRA BOLA (E=12 CM) & M3 & 85,42 & 34,20 & $2.921,39$ & 21,36 & 1 \\
\hline 6 & CAMA DE ARENA (2 CM) & M3 & 14,24 & 31,80 & 452,73 & 3,56 & 1 \\
\hline 7 & HORMIGON DE REPLANTILLO FC $=140 \mathrm{KG} / \mathrm{CM} 2 \mathrm{E}=5 \mathrm{CM}$ & M3 & 148,45 & 167,89 & $24.922,93$ & 37,11 & 7 \\
\hline 9 & ENCOFRADO DE ZAPATAS & $\mathrm{M} 2$ & 954,84 & 25,63 & $24.472,55$ & 238,71 & 10 \\
\hline 10 & ACERO DE REFUERZO EN BARRAS FY=4200 KG/CM2 (ZAPATAS) & KG & $47.984,03$ & $\begin{array}{cc}2,0,64 \\
1,64\end{array}$ & $78.693,82$ & $11.996,01$ & 15 \\
\hline 11 & HORMIGON DE MUROS FC $=210 \mathrm{KG} / \mathrm{CM} 2$ & M3 & $1.242,97$ & 204,62 & $254.336,93$ & 310,74 & 21 \\
\hline 12 & ENCOFRADO DE MUROS & M2 & $34.504,88$ & 39,16 & $1.351 .211,10$ & $8.626,22$ & 28 \\
\hline 13 & ACERO DE REFUERZO EN BARRAS FY=4200 KG/CM2 (MUROS) & KG & $157.891,36$ & 1,60 & $252.626,18$ & $39.472,84$ & 22 \\
\hline 14 & HORMIGON DE VIGAS DE ENTREPISO FC $=210 \mathrm{KG} / \mathrm{CM} 2$ & M3 & 265,73 & 218,41 & $58.037,65$ & 66,43 & 13 \\
\hline 15 & ENCOFRADO DE VIGAS DE ENTREPISO & $\mathrm{M} 2$ & $1.044,00$ & 40,92 & $42.720,48$ & 261,00 & 11 \\
\hline 16 & ACERO DE REFUERZO EN BARRAS FY $=4200 \mathrm{KG} / \mathrm{CM} 2$ (VIGAS ENTREPISO) & KG & $50.235,92$ & 1,64 & $82.386,92$ & $12.558,98$ & 16 \\
\hline 17 & HORMIGON DE LOSAS DE ENTREPISO FC $=210 \mathrm{KG} / \mathrm{CM} 2$ & $\mathrm{M} 3$ & 672,97 & 206,89 & $139.231,18$ & 168,24 & 14 \\
\hline 18 & ENCOFRADO DE LOSAS DE ENTREPISO & $\mathrm{M} 2$ & $4.435,28$ & 39,64 & $175.814,50$ & $1.108,82$ & 12 \\
\hline 20 & HORMIGON DE CAMASTROS FC $=210 \mathrm{KG} / \mathrm{CM} 2$ & $\mathrm{M} 3$ & 211,68 & 221,44 & $46.874,42$ & 52,92 & 11 \\
\hline 21 & ENCOFRADO DE LOSAS DE CAMASTROS & $\mathrm{M} 2$ & $2.817,72$ & 39,13 & $110.257,38$ & 704,43 & 7 \\
\hline 22 & ACERO DE REFUERZO EN BARRAS FY=4200 KG/CM2 (CAMASTROS) & KG & $21.097,44$ & 1,66 & $35.021,75$ & $5.274,36$ & 7 \\
\hline 23 & HORMIGON DE CONTRAPISO FC=210 KG/CM2 & $\mathrm{M} 3$ & 106,74 & 199,30 & $21.273,28$ & 26,69 & 2 \\
\hline 24 & MALLA ELECTROSOLDADA R-131 (5 MM / 150 MM) PARA CONTRAPISO & M2 & 711,60 & 7,19 & $5.116,40$ & 177,90 & 2 \\
\hline 25 & POLIETILENO & $\mathrm{M} 2$ & 711,84 & 2,45 & $1.744,01$ & 177,96 & 2 \\
\hline 26 & HORMIGON DE MURO DE CERRAMIENTO FC=210 KG/CM2 & $\mathrm{M} 3$ & 526,80 & 247,84 & $130.562,11$ & 131,70 & 26 \\
\hline 27 & ENCOFRADO DE MUROS DE CERRAMIENTO & M2 & $2.715,44$ & 43,44 & $117.958,71$ & 678,86 & 28 \\
\hline \multirow[t]{2}{*}{28} & ACERO DE REFUERZO EN BARRAS FY=4200 KG/CM2 (MUROS CERRAMIENTO) & KG & $37.528,92$ & 1,68 & $63.048,59$ & $9.382,23$ & 23 \\
\hline & & & & & $3.307 .880,28$ & & \\
\hline
\end{tabular}


TABLA III. PRESUPUESTO DE LA ESTRUCTURA DE HORMIGÓN ARMADO DE LOS 4 PABELLONES CON EL MÉTODO EN CADENA O EN SERIE. Fuente: [6].

\begin{tabular}{|c|c|c|c|c|c|c|c|}
\hline RUBRO & DESCRIPCION & UNIDAD & CANTIDAD & $\begin{array}{l}\text { PRECIO } \\
\text { UNITARIO }\end{array}$ & $\begin{array}{c}\text { PRECIO } \\
\text { TOTAL }\end{array}$ & \begin{tabular}{|c|} 
CANTIDAD 1 \\
PABELLON \\
(ALA1 Y ALA2)
\end{tabular} & $\begin{array}{c}\text { NUMERO } \\
\text { DE DIAS }\end{array}$ \\
\hline 1 & EXCAVACION SIN CLASIFICAR & M3 & $2.298,37$ & 1,86 & $4.274,96$ & 574,59 & 2 \\
\hline 2 & DESALOJO DE LAS EXCAVACIONES DISTANCIA $10 \mathrm{KM}$ & M3-KM & $22.983,68$ & 0,48 & $11.032,17$ & $5.745,92$ & 2 \\
\hline 3 & RELLENO CON MATERIAL DE MEJORAMIENTO (INCLUIDO TRANSPORTE) & M3 & $1.189,74$ & 15,75 & $18.738,46$ & 297,44 & 1 \\
\hline 4 & RELLENO CON SUELO CEMENTO (INCLUIDO TRANSPORTE) & M3 & 320,54 & 32,29 & $10.350,37$ & 80,14 & 1 \\
\hline 5 & EMPEDRADO CON PIEDRA BOLA (E=12 CM) & $\mathrm{M} 3$ & 85,42 & 35,94 & $3.070,02$ & 21,36 & 1 \\
\hline 6 & CAMA DE ARENA (2 CM) & M3 & 14,24 & 33,42 & 475,79 & 3,56 & 1 \\
\hline 7 & HORMIGON DE REPLANTILLO FC=140 KG/CM2 E=5CM & $\mathrm{M} 3$ & 148,45 & 176,43 & $26.190,68$ & 37,11 & 7 \\
\hline 9 & ENCOFRADO DE ZAPATAS & M2 & 954,84 & 26,93 & $25.713,84$ & 238,71 & 10 \\
\hline 10 & ACERO DE REFUERZO EN BARRAS FY=4200 KG/CM2 (ZAPATAS) & KG & $47.984,03$ & 1,72 & $82.532,54$ & $11.996,01$ & 15 \\
\hline 11 & HORMIGON DE MUROS FC=210 KG/CM2 & $\mathrm{M} 3$ & $1.242,97$ & 215,03 & $267.276,27$ & 310,74 & 21 \\
\hline 12 & ENCOFRADO DE MUROS & M2 & $34.504,88$ & 24,16 & $833.637,90$ & $8.626,22$ & 28 \\
\hline 13 & ACERO DE REFUERZO EN BARRAS FY=4200 KG/CM2 (MUROS) & KG & $157.891,36$ & 1,69 & $266.836,40$ & $39.472,84$ & 22 \\
\hline 14 & HORMIGON DE VIGAS DE ENTREPISO FC=210 KG/CM2 & M3 & 265,73 & 229,51 & $60.987,23$ & 66,43 & 13 \\
\hline 15 & ENCOFRADO DE VIGAS DE ENTREPISO & M2 & $1.044,00$ & 26,00 & $27.144,00$ & 261,00 & 11 \\
\hline 16 & ACERO DE REFUERZO EN BARRAS FY=4200 KG/CM2 (VIGAS ENTREPISO) & KG & $50.235,92$ & 1,72 & $86.405,79$ & $12.558,98$ & 16 \\
\hline 17 & HORMIGON DE LOSAS DE ENTREPISO FC=210 KG/CM2 & $\mathrm{M} 3$ & 672,97 & 217,41 & $146.310,84$ & 168,24 & 14 \\
\hline 18 & ENCOFRADO DE LOSAS DE ENTREPISO & M2 & $4.435,28$ & 24,65 & $109.329,65$ & $1.108,82$ & 12 \\
\hline 20 & HORMIGON DE CAMASTROS FC=210 KG/CM2 & M3 & 211,68 & 232,70 & $49.257,94$ & 52,92 & 11 \\
\hline 21 & ENCOFRADO DE LOSAS DE CAMASTROS & M2 & $2.817,72$ & 24,12 & $67.963,41$ & 704,43 & 7 \\
\hline 22 & ACERO DE REFUERZO EN BARRAS FY=4200 KG/CM2 (CAMASTROS) & KG & $21.097,44$ & 1,75 & $36.920,52$ & $5.274,36$ & 7 \\
\hline 23 & HORMIGON DE CONTRAPISO FC=210 KG/CM2 & $\mathrm{M} 3$ & 106,74 & 209,44 & $22.355,63$ & 26,69 & 2 \\
\hline 24 & MALLA ELECTROSOLDADA R-131 (5 MM / 150 MM) PARA CONTRAPISO & $\mathrm{M} 2$ & 711,60 & 7,55 & $5.372,58$ & 177,90 & 2 \\
\hline 25 & POLIETILENO & $\mathrm{M} 2$ & 711,84 & 2,58 & $1.836,55$ & 177,96 & 2 \\
\hline 26 & HORMIGON DE MURO DE CERRAMIENTO FC=210 KG/CM2 & M3 & 526,80 & 260,44 & $137.199,79$ & 131,70 & 26 \\
\hline 27 & ENCOFRADO DE MUROS DE CERRAMIENTO & M2 & $2.715,44$ & 28,64 & $77.770,20$ & 678,86 & 28 \\
\hline \multirow[t]{2}{*}{28} & ACERO DE REFUERZO EN BARRAS FY=4200 KG/CM2 (MUROS CERRAMIENTO) & KG & $37.528,92$ & 1,76 & $66.050,90$ & $9.382,23$ & 23 \\
\hline & & & & & $2.703 .169,35$ & & \\
\hline
\end{tabular}

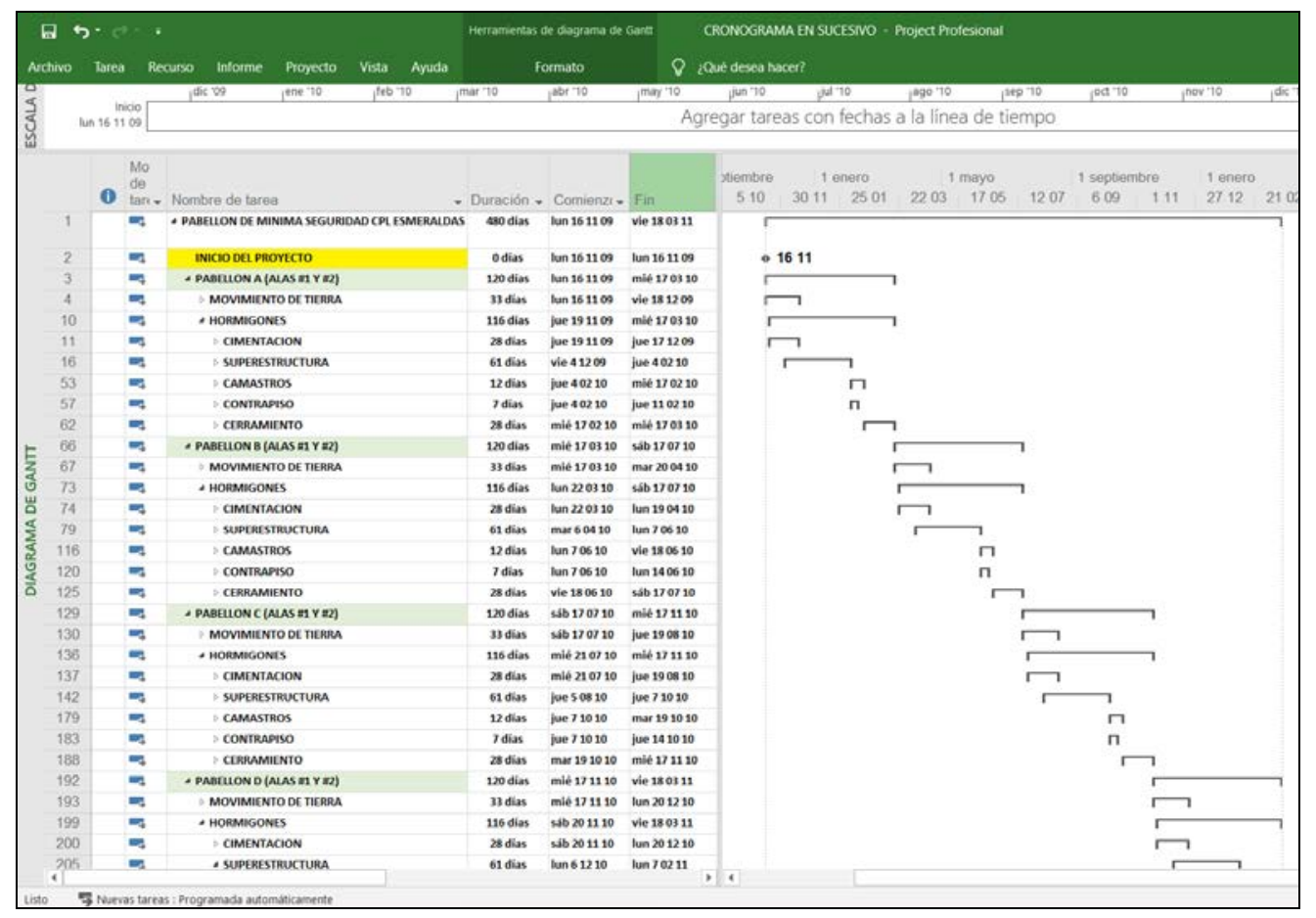

Fig. 1 Cronograma de la construcción de los 4 pabellones por medio del método sucesivo en el programa MS Project.

Fuente: [6]. 


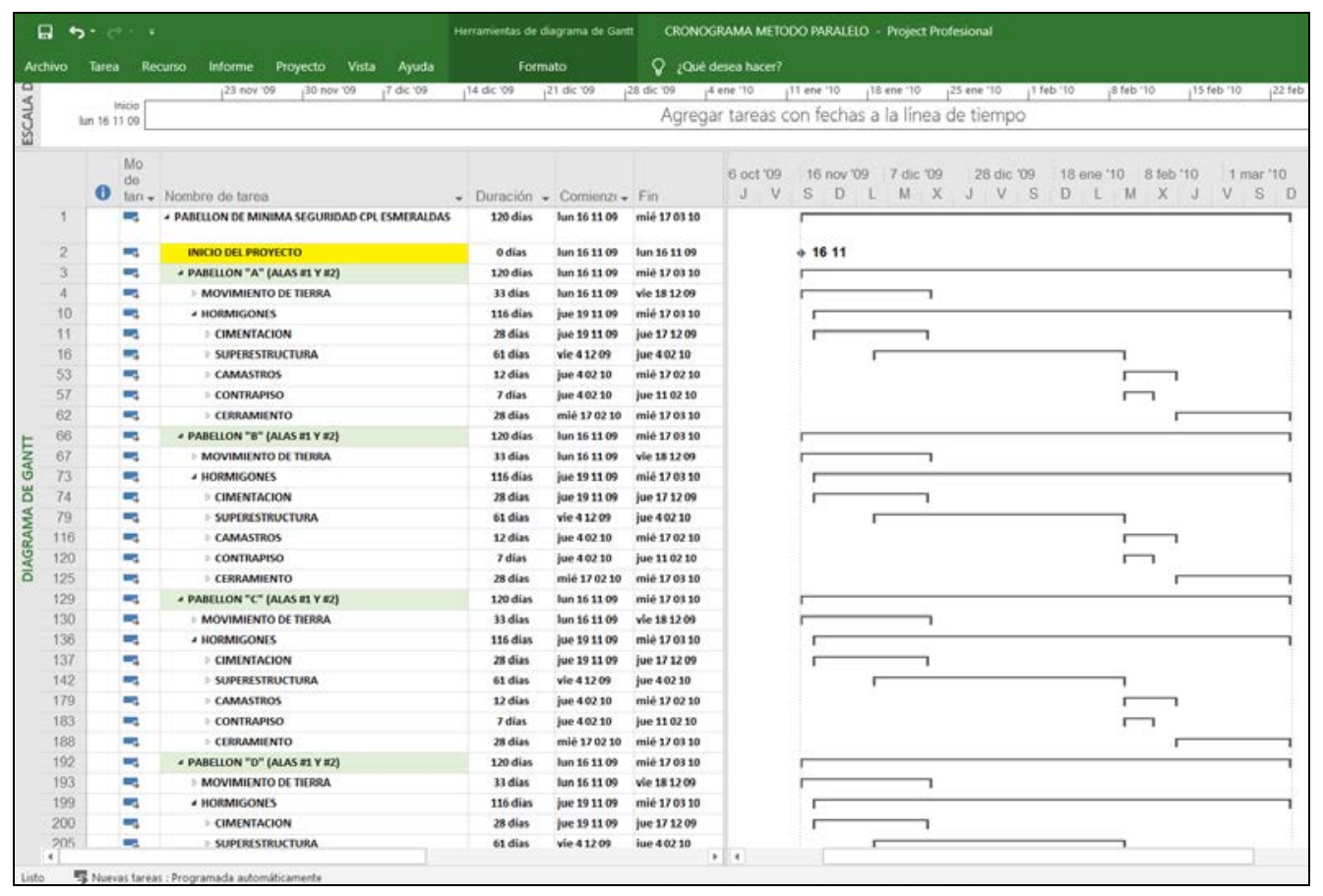

Fig. 2 Cronograma de la construcción de los 4 pabellones por medio del método paralelo o simultáneo en el programa MS Project. Fuente: [6].

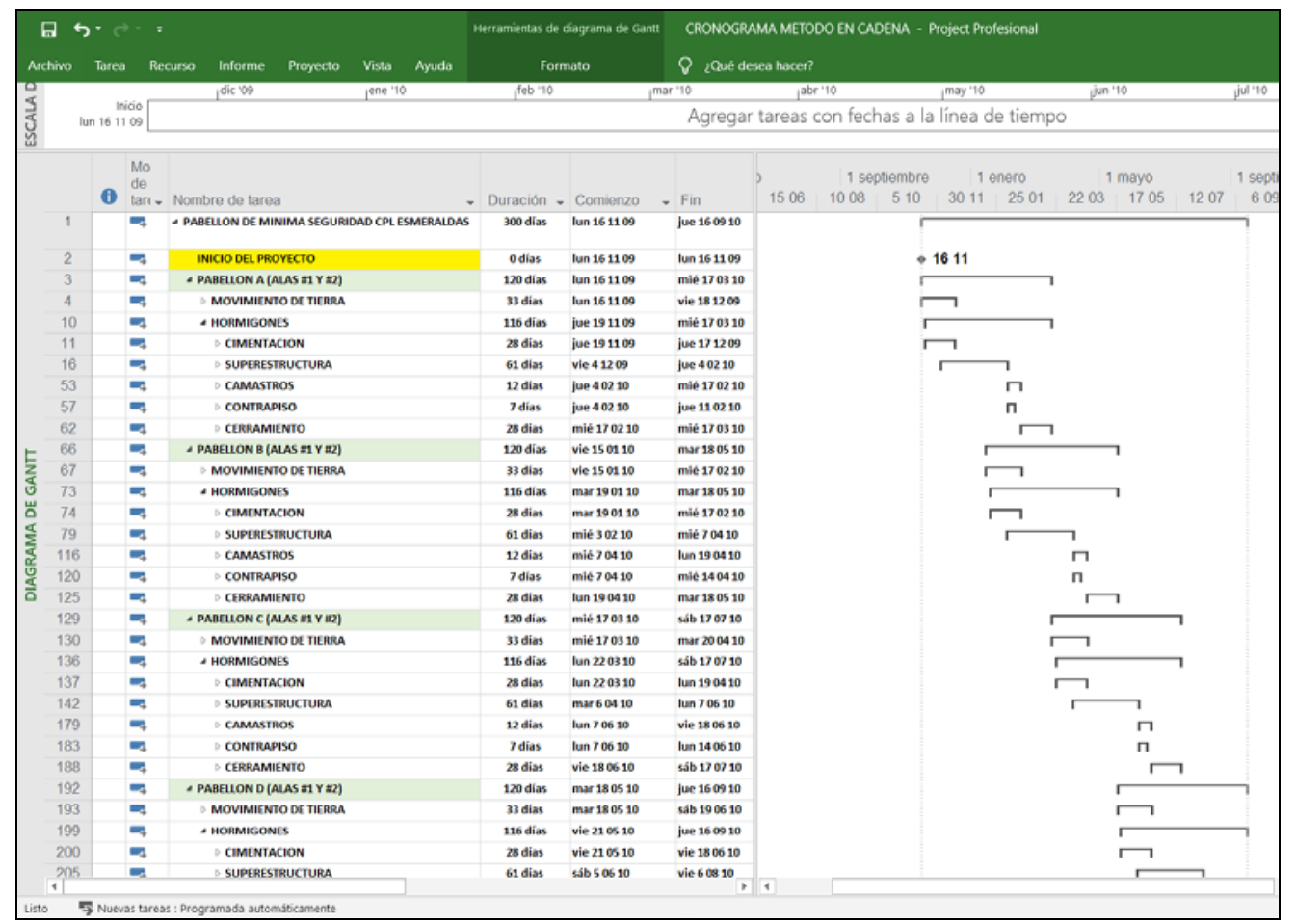

Fig. 3 Cronograma de la construcción de los 4 pabellones por medio del método en cadena o en serie en el programa MS Project. Fuente: [6]. 


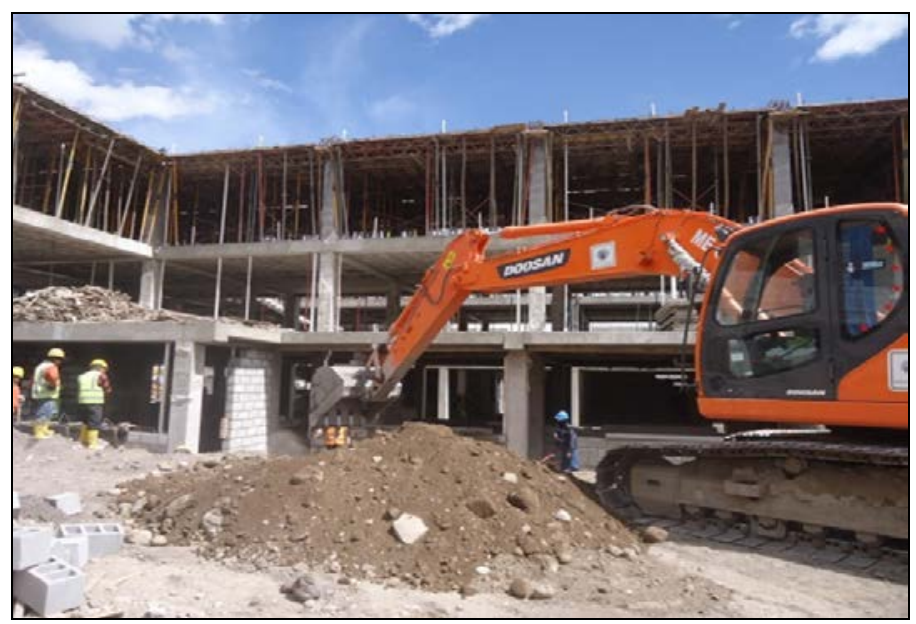

Fig. 10 Foto del rubro de excavación sin clasificar. Fuente: Propia.

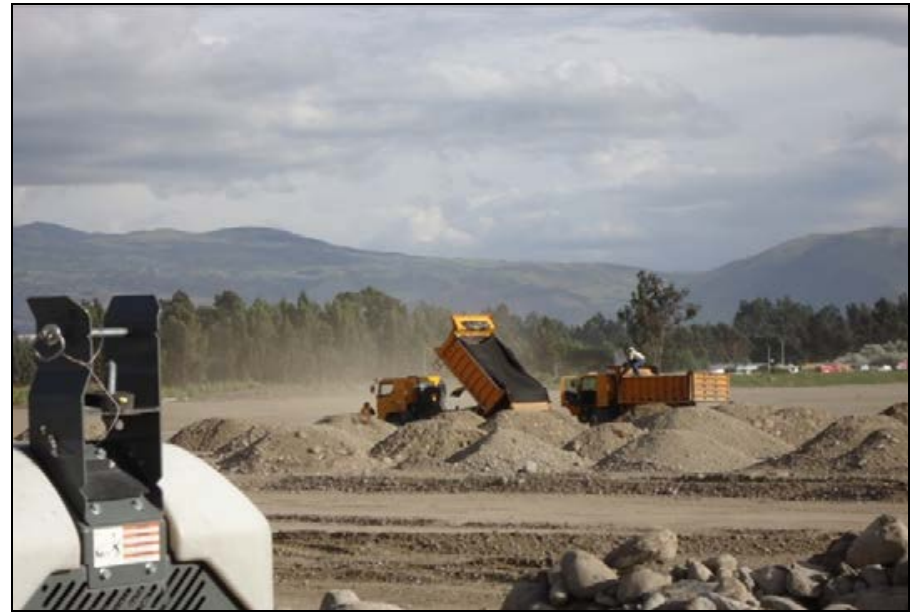

Fig. 11 Foto del rubro de relleno con material de mejoramiento (inc. transporte). Fuente: Propia.

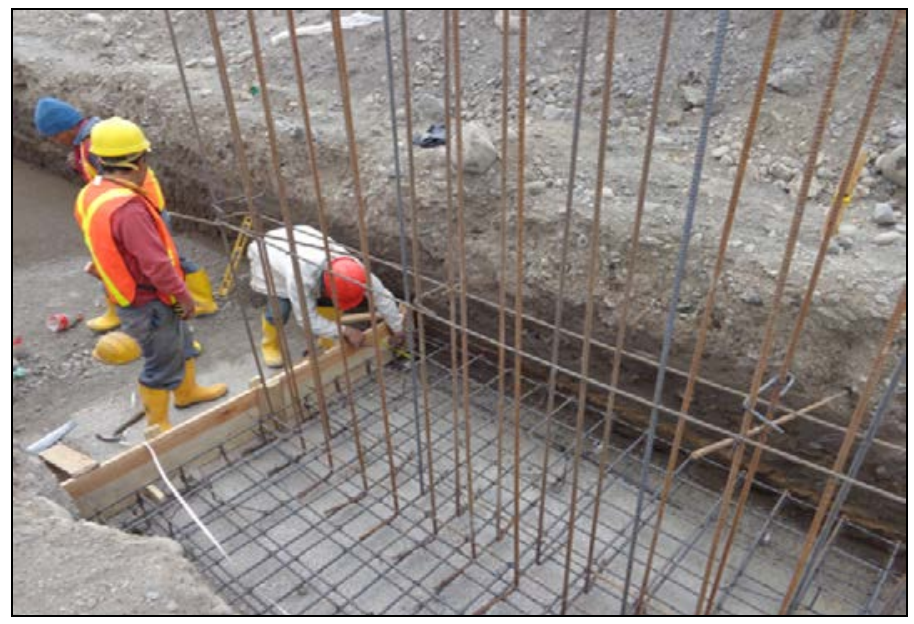

Fig. 12 Foto del rubro de acero de refuerzo en varillas corrugadas fy=4200 kg/cm2 para zapata. Fuente: Propia. 


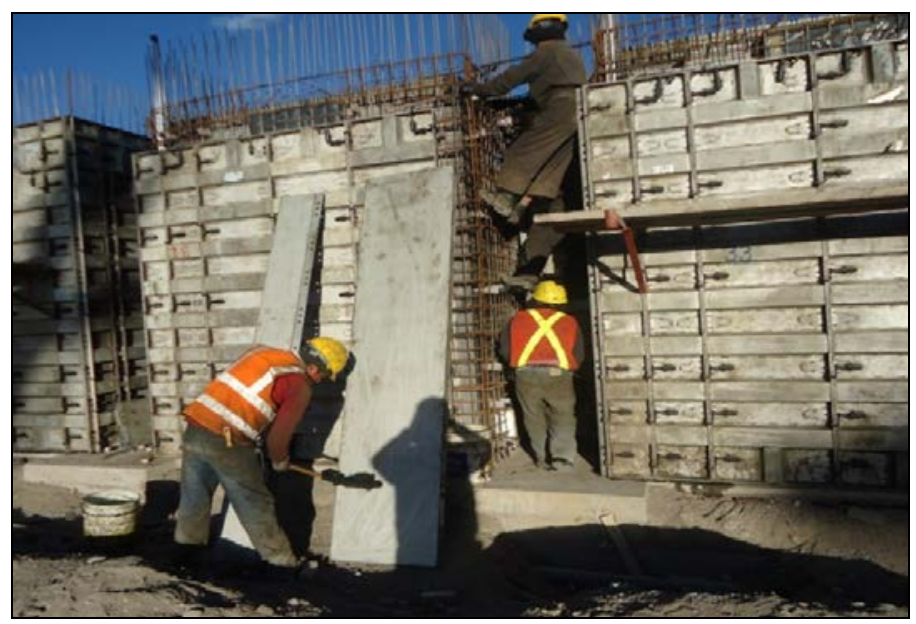

Fig. 13 Foto del rubro encofrado de muros estructurales. Fuente: Propia.

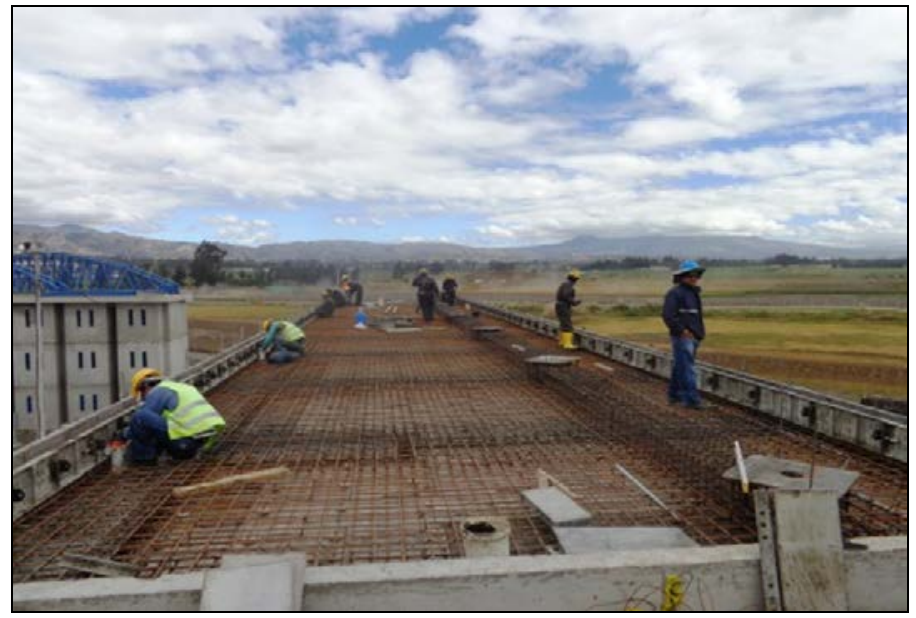

Fig. 13 Foto del rubro de malla electrosoldada en losas. Fuente: Propia.

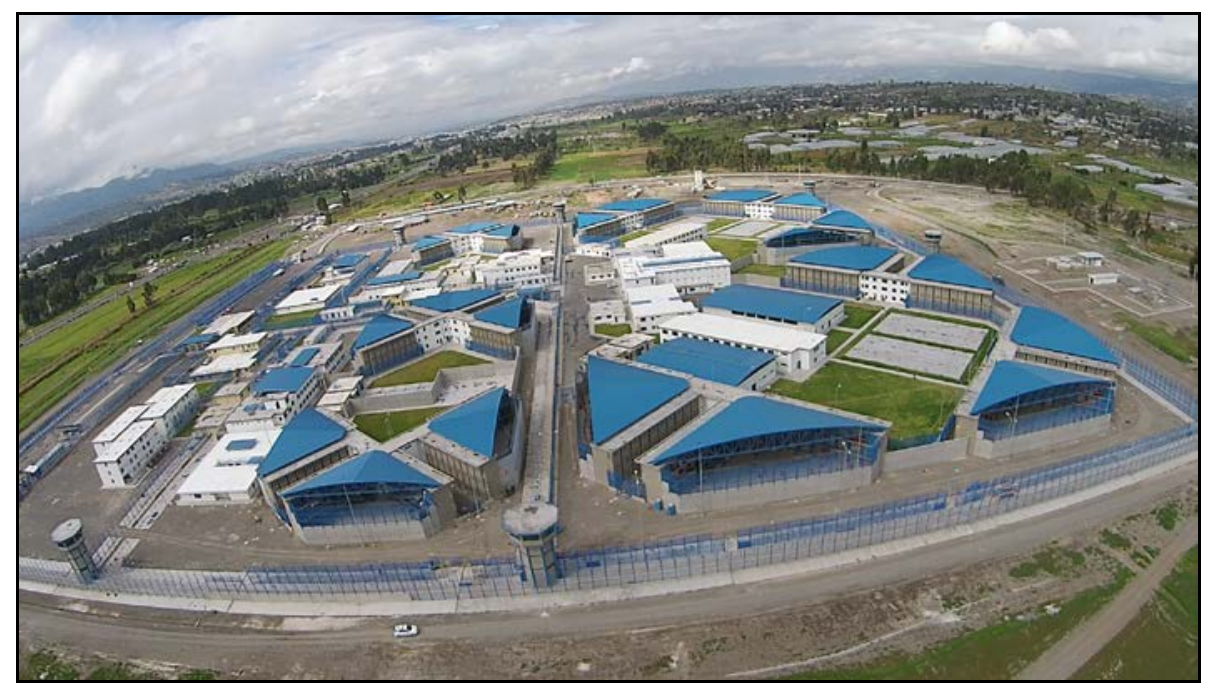

Fig. 14 Imagen de referencia del Centro de Rehabilitación Social Regional Sierra Centro Norte (CRS) de Cotopaxi. Fuente: Propia. 